\title{
Transcriptional Control of Behavior: Engrailed Knock-Out Changes Cockroach Escape Trajectories
}

\author{
David Booth, ${ }^{1}$ Bruno Marie, ${ }^{2}$ Paolo Domenici, ${ }^{3}$ Jonathan M. Blagburn, ${ }^{2}$ and Jonathan P. Bacon ${ }^{1}$ \\ ${ }^{1}$ Sussex Centre for Neuroscience, School of Life Sciences, University of Sussex, Falmer, Brighton BN1 9QG, United Kingdom, ${ }^{2}$ Institute of Neurobiology, \\ University of Puerto Rico, San Juan, Puerto Rico 00901, and ${ }^{3}$ Consiglio Nazionale delle Ricerche-Istituto Ambiente Marino Costiero/International Marine \\ Centre Localita Sa Mardini, 09072 Torregrande (Oristano), Italy
}

The cerci of the cockroach are covered with identified sensory hairs that detect air movements. The sensory neurons that innervate these hairs synapse with giant interneurons in the terminal ganglion that in turn synapse with interneurons and leg motor neurons in thoracic ganglia. This neural circuit mediates the animal's escape behavior. The transcription factor Engrailed (En) is expressed only in the medially born sensory neurons, which suggested that it could work as a positional determinant of sensory neuron identity. Previously, we used double-stranded RNA interference to abolish En expression and found that the axonal arborization and synaptic outputs of an identified En-positive sensory neuron changed so that it came to resemble a nearby En-negative cell, which was itself unaffected. We thus demonstrated directly that En controls synaptic choice, as well as axon projections. Is escape behavior affected as a result of this miswiring? We showed recently that adult cockroaches keep each escape unpredictable by running along one of a set of preferred escape trajectories (ETs) at fixed angles from the direction of the threatening stimulus. The probability of selecting a particular ET is influenced by wind direction. In this present study, we show that early instar juvenile cockroaches also use those same ETs. En knock-out significantly perturbs the animals' perception of posterior wind, altering the choice of ETs to one more appropriate for anterior wind. This is the first time that it has been shown that knock-out of a transcription factor controlling synaptic connectivity can alter the perception of a directional stimulus.

\section{Introduction}

The engrailed (en) gene codes for a homeodomain transcription factor that was originally characterized as playing a key role during segmentation in Drosophila (Nüsslein-Volhard and Wieschaus, 1980). En has subsequently been shown to have an additional conserved role in neuronal development in both insects and vertebrates (Condron et al., 1994; Friedman and O'Leary, 1996; Logan et al., 1996; Lundell et al., 1996; Baader et al., 1999; Saueressig et al., 1999; Siegler and Jia, 1999; Joly et al., 2007). Homologs of engrailed have since been cloned from a wide variety of triploblastic animals, including the two paralogs of the cockroach Periplaneta americana: $\mathrm{Pa}-\mathrm{En} 1$ and Pa-En2 (Marie and Bacon, 2000).

In the cockroach, En is expressed in medial sensory neurons of the cerci, the posterior sensory appendages that bear windsensitive hairs (Blagburn et al., 1995). These wind-sensory neurons synapse with giant interneurons (GIs) in the terminal ganglion, which in turn synapse with interneurons and leg motor neurons in thoracic ganglia. This extensively characterized neural

Received March 23, 2009; accepted April 25, 2009.

This study was supported by a Biotechnology and Biological Sciences Research Council studentship (D.B.) and grant (J.P.B.), grants from the Consiglio Nazionale delle Ricerche (P.D.), National Institutes of Health Support of Competitive Research Grant SC2 NS065384 (J.M.B.), and Research Centers in Minority Institutions Grant G12 RR03051 (J.M.B., B.M.)

Correspondence should be addressed to Dr. Jonathan M. Blagburn, Institute of Neurobiology, 201 Boulevard del Valle, San Juan, Puerto Rico 00901. E-mail: jonathan.blagburn@upr.edu or jblagburn@gmail.com.

DOI:10.1523/JNEUROSCI.1374-09.2009

Copyright $\odot 2009$ Society for Neuroscience $\quad 0270-6474 / 09 / 297181-10 \$ 15.00 / 0$ circuit mediates the cockroach's escape behavior (Westin et al., 1977; Blagburn et al., 1984; Comer and Dowd, 1987; Ritzmann and Pollack, 1988; Kolton and Camhi, 1995; Levi and Camhi, 2000a).

Previously, we abolished En expression by using doublestranded RNA interference (dsRNAi). We found that an identified medial neuron $(6 \mathrm{~m})$ then forms an arborization typical of its lateral, normally En-negative, neighbor. En knock-out also alters the pattern of monosynaptic connections made by the neuron to target GIs (Marie et al., 2000). Subsequent work showed that En controls axon arborization independently of its control of synaptic specificity (Marie et al., 2002) and that both paralogs control axonal guidance whereas Pa-En1 alone is responsible for determining synaptic target recognition (Marie and Blagburn, 2003). Medial sensory neurons respond preferentially to wind from the rear of the animal (Dagan and Camhi, 1979; Dagan and Volman, 1982), so we hypothesized that the animal's directional response to wind would be affected by En knock-out. It remains an open question whether these cellular alterations in the synaptic connectivity of $6 \mathrm{~m}$ also take place in the other medial neurons and whether they are sufficient to alter significantly the escape behavior of the animal as a whole.

We showed recently that adult cockroaches are able to keep predators "guessing," making each escape unpredictable by running along one of a set of preferred escape trajectories (ETs) at fixed angles from the direction of the threatening stimulus. The probability of selecting a particular ET is influenced by wind direction (Domenici et al., 2008). In this present study, we show 
that En knock-out significantly perturbs the animals' perception of posterior wind. This is manifest not as a change in turn angle but as an alteration in the selection of ETs, so as to follow a strategy more suited to anterior wind. To our knowledge, this is the first time that it has been shown that knock-out of a transcription factor controlling synaptic connectivity can alter the perception of a directional stimulus.

\section{Materials and Methods}

dsRNA preparation. Aliquots of a 1:1 mixture of pa-en 1 and pa-en 2 dsRNAs were prepared as described previously (Marie et al., 2000) and stored at $-80^{\circ} \mathrm{C}$ before use.

Injection of dsRNA and controls. Newly hatched first-instar cockroaches were subjected to a dehydrating regimen for $2-3 \mathrm{~h}$ in a warm $\left(37^{\circ} \mathrm{C}\right)$ incubator at very low humidity. This was found to be critical for successful injection because hemolymph pressure in fully hydrated nymphs prevented reliable uptake of the injected fluid through the cercal lumen. After dehydration, the nymphs were transferred in groups of three to small, lidded Petri dishes held in a larger plastic container in which the floor was covered by desiccant silica gel. They were anesthetized with $\mathrm{CO}_{2}$ for $1 \mathrm{~min}$ and then positioned dorsal side up on a fine wire mesh with a flow of $\mathrm{CO}_{2}$ from below to maintain anesthesia.

The terminal segment of the cercus was broken off using fine forceps and $\sim 150 \mathrm{nl}$ of the dsRNA mixture injected into the cercal lumen. After our dehydration regimen, this volume was taken up readily from the cut end of the cercus and there was no need to inject it forcibly. Two injections were given $1 \mathrm{~h}$ apart to each animal because there is evidence that the dsRNA from the first injection is primarily taken up by hemocytes (Marie et al., 2000). Sham-operated control animals were handled in exactly the same manner but the injection consisted of carrier buffer alone (20 mм Tris and $150 \mathrm{~mm} \mathrm{NaCl}, \mathrm{pH} 7.8)$. Other controls were not injected. Once treated, the insects were transferred to rearing containers kept at $25-28^{\circ} \mathrm{C}$ with food (cat biscuits) and water available ad libitum.

En immunocytochemistry. Second- and third-instar animals were placed in saline, and the dorsal tergites and gut were removed. The distal two segments of the cerci were cut off, and fixative (4\% paraformaldehyde in $0.075 \mathrm{M}$ PBS) was added to the dissection chamber. The dorsal side of each cercus was then cut off, exposing the interior. Tracheae, the cercal nerves, connective tissue, and as many fat body cells as possible were removed with fine forceps to improve access and visibility of the sensory neurons. Fat body cells in particular tend to show strong granular avidin binding in the cytoplasm. Animals were fixed for $1 \mathrm{~h}$ at $4^{\circ} \mathrm{C}$, before washing thoroughly in PBS. After a preincubation in normal horse serum in PBS plus $0.3 \%$ Triton X-100 (PBST) for $30 \mathrm{~min}$, monoclonal 4D9 anti En antibody, obtained from the Developmental Studies Hybridoma Bank (University of Iowa, Iowa City, IA), was applied at a dilution of 1:20 in PBST for $15-20 \mathrm{~h}$ at $4^{\circ} \mathrm{C}$. After three 10 -min washes, biotinylated horse anti-mouse antibody (Vector Laboratories) was applied at a dilution of 1:200 for $1 \mathrm{~h}$ at room temperature, and the tissue was again washed three times. After incubation in avidin-peroxidase complex (Vector Laboratories) for $1 \mathrm{~h}$ at room temperature, the tissue was washed, a solution of $0.1 \%$ diaminobenzidine with $0.03 \%$ hydrogen peroxide was added to the wells, and the reaction was allowed to proceed for $20 \mathrm{~min}$. The specimens were washed in PBS and then cleared and mounted in $70 \%$ glycerol. Images were captured with a Zeiss Axiocam CCD camera. Cerci from at least three animals were examined for each treatment.
Behavioral trials. Animals were placed in the arena in a darkened room in groups of five for periods of up to $90 \mathrm{~min}$. The responses of shamoperated controls were not significantly different from those of unmanipulated animals (Watson's $\mathrm{U}^{2}$ test, $p>0.05$ ); for this reason, the data were amalgamated and are presented below as "controls."

Experiments were performed to record as many trials as possible over the full range of wind stimulus directions for each category of insect. Wind stimuli were delivered only when the insects passed over a line on the arena floor, with which the wind stimulus nozzle was aligned. The line was sufficiently far from the glass walls of the arena to avoid escape behavior being affected by the insects' natural thigmotaxis. The position of the wind stimulus in relation to the room layout was randomized between groups of trials.

A subset of the insects used in behavioral trials in the second instar were allowed to develop on to the third instar and were then given an additional set of trials. The aim of this experiment was to determine whether there was any recovery of normal escape behavior in the treated insects after the augmentation of the second-instar sensilla with their third-instar counterparts.

Trials were performed in a circular arena $15 \mathrm{~cm}$ in diameter with 3 -cm-high walls in a darkened room under far-red (>675 nm) illumination provided by an array of light-emitting diodes. The wind stimulus was delivered through a 6-mm-diameter tube positioned $10 \mathrm{~mm}$ above the arena floor at an angle of $45^{\circ}$ to the horizontal, using a modified smaller version of the loudspeaker-cone-driven wind stimulus machine described previously (Stern et al., 1997). Peak wind velocity of the stimuli was $\sim 0.1 \mathrm{~ms}^{-1}$ as measured using a miniature hot-wire anemometer. All trials were captured on video at 50 frames/s using a monochrome CCD camera (Sony AVC-D7CE) and video cassette recorder (Panasonic 999).

Wind stimulus directions were measured with respect to the animal's position immediately before the onset of the wind stimulus. Because no differences were found in escapes when the cockroaches were stimulated 

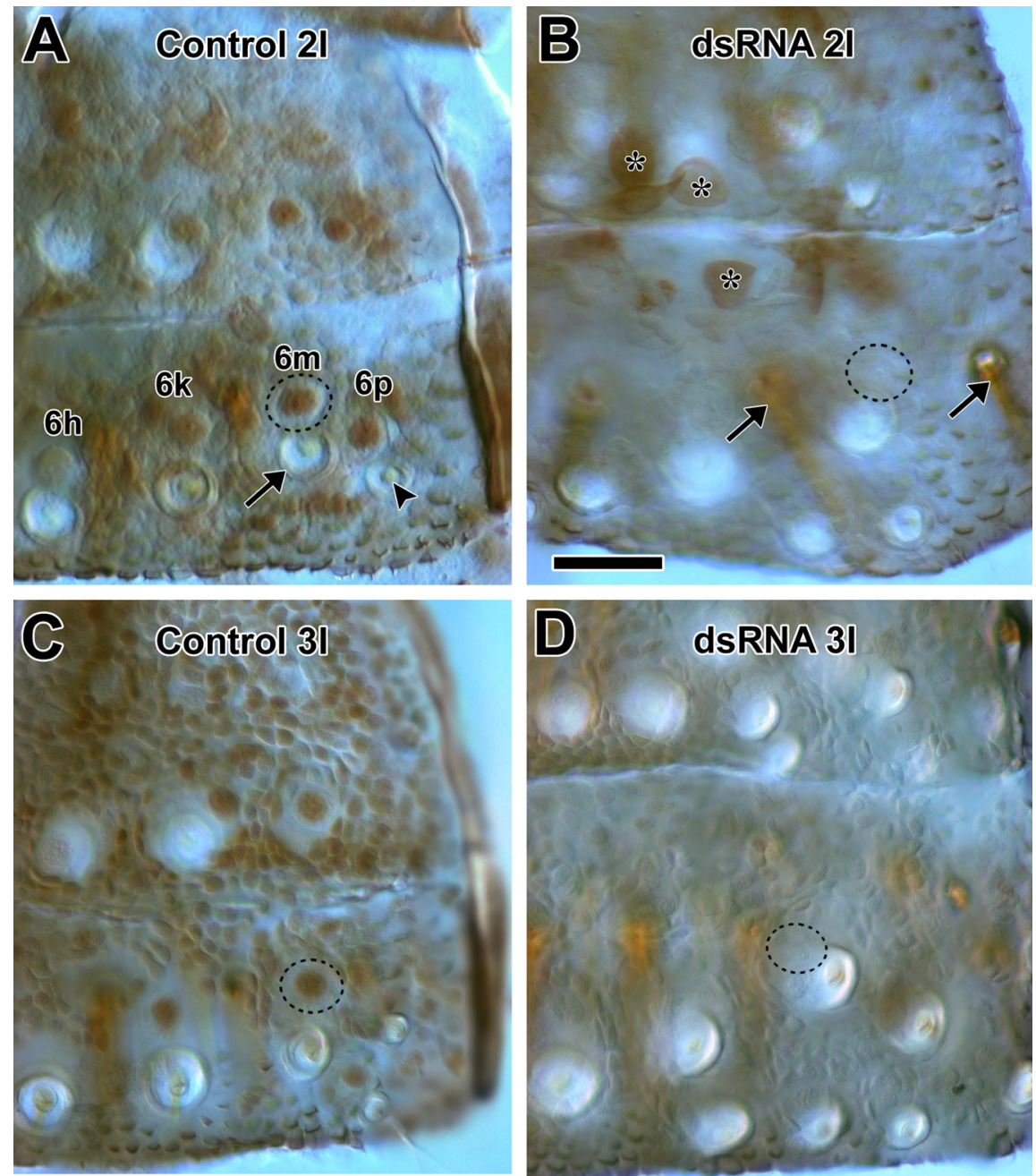

Figure 2. Engrailed dsRNA treatment abolishes cercal En immunostaining in second- and third-instar cerci. Preparations are representative of at least three animals for each treatment. $A$, Medial portion of second-instar segments 6 and 7 , showing En-immunoreactive nuclei of epidermal cells and neurons. Identified sensory neuron $6 \mathrm{~h}$ express En weakly, $6 \mathrm{k}$ expresses En at an intermediate level, and $6 \mathrm{~m}$ (dashed ellipse) and $6 \mathrm{p}$ express it strongly. The arrow and arrowhead mark the sockets of filiform hairs $6 \mathrm{~m}$ and $6 \mathrm{p}$, respectively. $\boldsymbol{B}$, Similar region from an animal treated with dsRNA in the first instar; medial neurons of segments 6 and $7 \mathrm{no}$ longer express En. A dashed ellipse indicates the soma of $6 \mathrm{~m}$. Arrows indicate out-of-focus bristle hairs, and asterisks indicate nonspecific staining of hemocytes and fat cells. C, Medial portion of third-instar segments 6 and 7, showing En-immunoreactive nuclei of epidermal cells and neurons. $\boldsymbol{D}$, Similar region from an animal, treated with dsRNA in the first instar, shows complete downregulation of En expression. Scale bar, $50 \mu \mathrm{m}$.

from either their left or right sides, escapes from left and right stimuli were pooled as if the stimuli were always from the right. Thus, wind directions spanned $0-180^{\circ}$, in which head-on stimuli were designated as $0^{\circ}$ and posterior stimuli corresponded to $180^{\circ}$. Escape turn angles were defined as the angle between the direction of motion at the end of the turning component of the escape and the initial heading of the animal (Domenici et al., 2008). ETs were defined as the angle between the escape turn and the direction of the stimulus. Therefore, ETs in a direction directly away from the stimulus were at $180^{\circ}$. ETs $>180^{\circ}$ indicated that animals had overturned beyond the direction of direct posterior stimulation. ETs potentially spanned $360^{\circ}$ and were treated as a circular variable (Batschelet, 1981). Both wind direction and escape angle were computed by analyzing video stills of the escape trials using a custom analysis program (HeadAngle; Kappa Software). Angle resolution attributable to pixel size was $1.1^{\circ}$

Statistical methods. Statistical tests on circular data were performed using Oriana 2 (Kovach Computing Services) as previously described (Domenici et al., 2008). Curves based on mixtures of multiple Gaussian distributions, with the constraints of the same SD and equal peak spacing, were fitted to unbinned ET data using the maximum likelihood method [mle program; Darryl Holman, University of Washington, Seattle, WA (Holman, 2003)]. The program evaluated possible mixtures of from 1 to 10 Gaussian curves (of equal width and spacing), and the best-fitting curve was chosen as being that with the highest corrected Akaike Information Criterion (AICc). The AICc balances a maximization of the likelihood against the minimization of the number of parameters, thus avoiding overfitting (Akaike, 1974; Hurvich and Tsai, 1989). The Akaike weight is directly derived from the AICc and gives the probability of that chosen model being the correct one compared with the alternatives. This approach does not rule out the possibility that more complex mixtures with Gaussians of variable width and spacing could more accurately describe the data; however, such models have many more parameters and are thus likely to have lower AICcs. These bestfit distributions were tested against binned experimental data using the $\chi^{2}$ test. Finally, peaks in each multimodal distribution were labeled as such if they contributed at least $5 \%$ to the best fit curve.

Estimation of GI receptive fields. The receptive fields of second-instar GI1-GI3 could not be measured directly because the electrophysiological recording setup necessitates submersion of the preparation in saline. Instead, receptive fields were estimated using methods described previously (Hill and Blagburn, 1998). We used action potential frequency data from the $3 \mathrm{M}$ hair of the first-instar cockroach (Dagan and Volman, 1982) and unitary monosynaptic EPSP amplitudes for the sensory afferents of segments 3-7 of the second-instar cercus (Thompson et al., 1992), along with measurements of the effect of En knock-out on the PSPs (Marie et al., 2000). The threshold for eliciting an action potential by ipsilateral or contralateral input was known for each GI; in most cases, the ipsilateral threshold was significantly higher than the contralateral, reflecting the different distances from the spike-initiating zone. The effective PSP amplitude was calculated by dividing the unitary PSP amplitude by the value of the spike threshold for that side of the neuron. Receptive fields for each GI were calculated by multiplying the afferent spike frequency at each angle by the effective PSP amplitude and then summing the result from each sensory afferent. Negative values were adjusted to zero. Although linear addition of the synaptic inputs does not take into account the complexities of temporal summation or homosynaptic facilitation, it nevertheless appears to give a reasonable approximation of the total depolarization in the GIs in response to a burst of sensory spikes (Hill and Blagburn, 1998). The resultant depolarization for each angle was converted to spike frequency using published data for GI maximal spiking rates (Kolton and Camhi, 1995) and was then plotted using polar coordinates.

\section{Results}

\section{The cercal system of the cockroach}

The first-instar cockroach nymph has only two functional windsensitive hairs per cercus, located on the proximal segment. On average, 37 second-instar neurons develop in the cercus shortly after hatching from the egg, so that, after molting to the second instar, there are 39 hairs per cercus (Thompson et al., 1992). The 
first instar lasts from 7 to $10 \mathrm{~d}$ (depending on environment) during which time the developing second-instar neurons grow into the terminal ganglion of the CNS, in which they form synapses with the GI system at approximately day 6 of development (Marie et al., 2002). Figure $1 A$ shows the position of the cerci in the second instar. Note that only those sensory neurons on the medial side of the cercus express En, whereas their lateral counterparts do not (Blagburn et al., 1995) (Fig. 1B). The second instar lasts for $\sim 20-30 \mathrm{~d}$; after molting again, the third-instar cercus has $\sim 55$ hairs.

\section{Persistence of En knock-out through to} the second and third instars

Previously we showed that the secondinstar neurons that are present in the firstinstar cercus before molting lose En expression after application of en dsRNA (Marie et al., 2000, 2002). For the present study, it was important to ascertain whether this knock-out persists in the second-instar animals and, indeed, whether it lasts into the third instar. We used the 4D9 monoclonal antibody to detect En proteins (both $\mathrm{Pa}$-En1 and $\mathrm{Pa}$-En2 paralogs) in flat-mounted cerci of secondand third-instar animals. Medial secondinstar neurons normally express En protein (Fig. 2A), but this expression is abolished after dsRNA application in the first instar, at least $8 \mathrm{~d}$ earlier (Fig. $2 \mathrm{~B}$ ). Medial third-instar cercal neurons also express En, in a pattern similar to earlier instars (Fig. 2C), and, significantly, this expression is still abolished after dsRNA treatment in the first instar (Fig. 2D), some $30 \mathrm{~d}$ after the dsRNAi treatment. The half-life of En protein is $\sim 30 \mathrm{~h}$ (Marie et al., 2002), which means that any En that is present in control second- and third-instar neurons must have been synthesized no more than $70 \mathrm{~h}$ earlier. This implies either that dsRNA and/or its inhibitory effects persist for many days after injection or, failing that, that the maintenance of en gene expression requires the presence of En protein, as is the case in Drosophila (Heemskerk et al., 1991).

\section{En knock-out alters escape turns}

Figure 3, $B$ and $D$, shows plots of escape turn angle versus wind angle for, respectively, second- and third-instar insects. Wind angles are measured with respect to the insect's initial body orientation, using the convention that $0^{\circ}$ represents a head-on stimulus and $180^{\circ}$ a stimulus from the rear of the animal. Data are amalgamated for left and right stimuli. The distribution of escape turns made by unoperated control animals were not significantly different from sham-operated controls (Watson's $U^{2}$ test, $p>$ 0.05 ), so for additional analyses, the results were pooled. Turn angles are measured as the deflection from the animal's initial heading, with positive angles representing turns to the right and negative values representing turns to the left. A bold dashed line representing an escape turn of $180^{\circ}$ away from the wind is drawn on each plot. Note that negative turn angles (turns to the left)
B

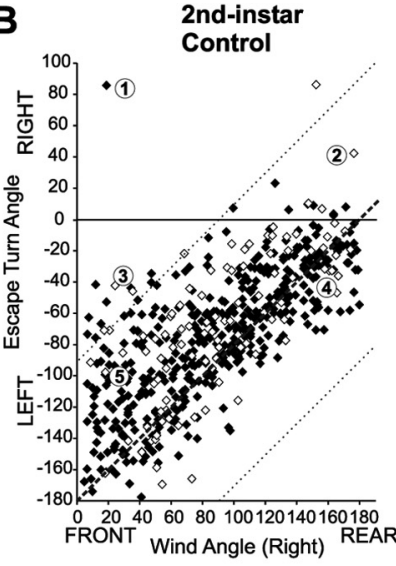

C $\begin{aligned} & \text { 2nd-insta } \\ & \text { dsRNA }\end{aligned}$
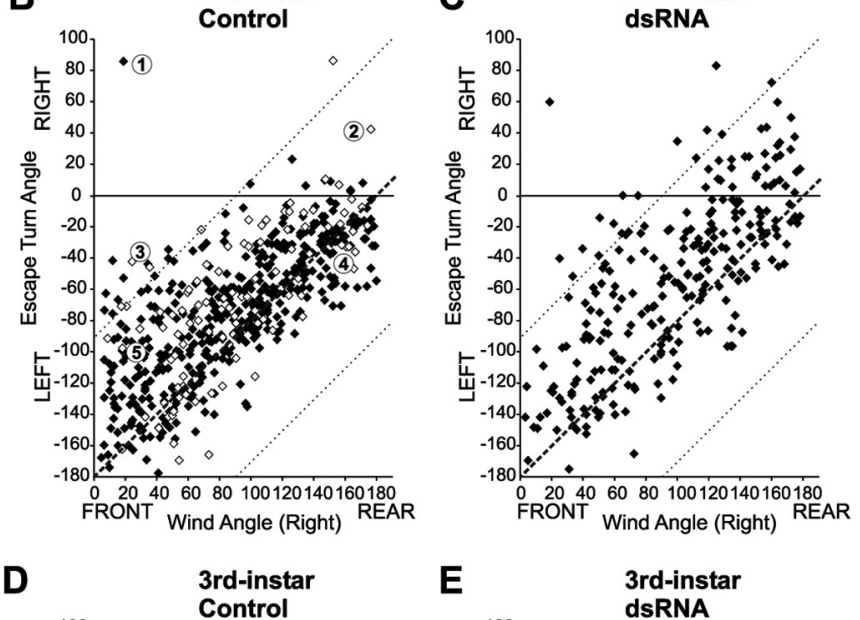

E $\begin{aligned} & \text { 3rd-instar } \\ & \text { dsRNA }\end{aligned}$
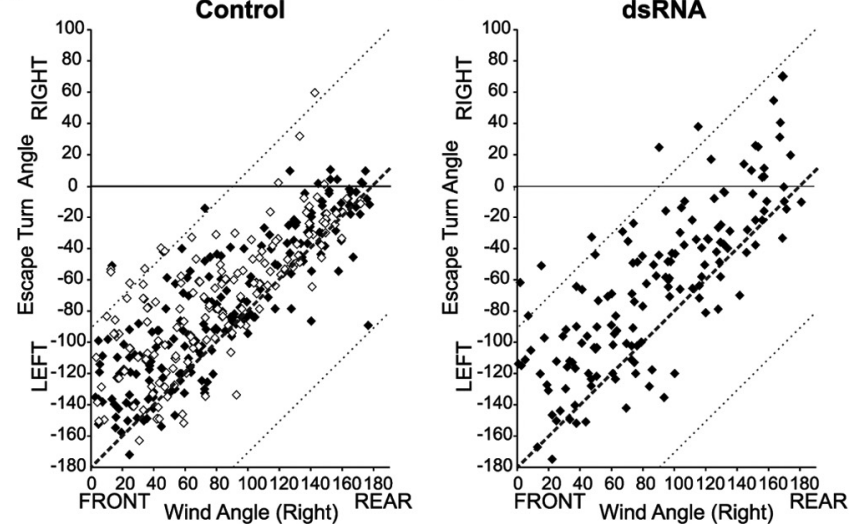

Figure 3. Engrailed knock-out alters escape turns. $\boldsymbol{A}$, Examples of escape turns plotted in $\boldsymbol{B}: 1$ and 2 are both toward turns, and 1 and 3 are examples of wrong turns. Gray arrow indicates stimulus direction. Wind angle (wind) at the time of stimulation and turns of $180^{\circ}$ away from the wind. The dotted lines indicate turns of $90^{\circ}$ relative to the stimulus. $\boldsymbol{B}$, Second-instar control (filled symbols) and sham-operated control (open symbols). Circled numbers indicate the positions of the escape turns shown in $\boldsymbol{A}$. $\boldsymbol{C}$, Second instar treated with dsRNA. D, Third-instar controls. $\boldsymbol{E}$, Third instar treated with dsRNA.

(Fig. 3A, 3-5) represent escape turns away from the stimulus direction (right), whereas turns "toward" the stimulus direction (Fig. 3A, 1 and 2) appear as positive turn angles. It should be noted that a small turn toward the stimulus may still result in the animal moving farther from the wind source (Fig. 3A,2) and so is not necessarily maladaptive. In contrast, turns that result in the animal taking a final heading that is $<90^{\circ}$ relative to the stimulus (outside the dotted lines on the scatter grams) are more likely to result in capture and were therefore termed "wrong" turns (Fig. $3 A, 1$ and 3 ).

The overall pattern of escape behavior shown in these plots is similar to that of other published data on early nymphs (Dagan and Volman, 1982; Stern et al., 1997), although the proportion of toward turns in the control groups is somewhat lower than in previous studies. This may be attributable, at least in part, to the way in which wind stimuli were presented. The stimulus nozzle remained fixed and was aligned precisely with a calibration mark drawn on the arena floor. Wind stimuli were delivered only when insects passed directly in line with the calibration mark, and, by implication, only walking insects were stimulated. In some of the previous studies, the wind stimulus apparatus was moved around the arena and it was more difficult to judge exactly when the insects were directly within the area of laminar flow from the nozzle.

En knock-out appears to alter the distribution of escape re- 

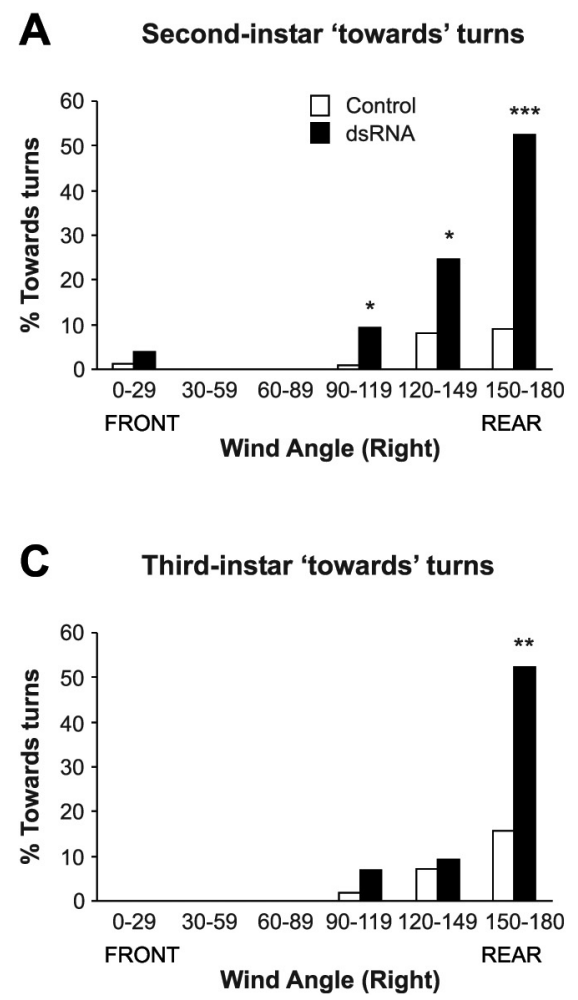

D

B

Second-instar 'wrong' turns

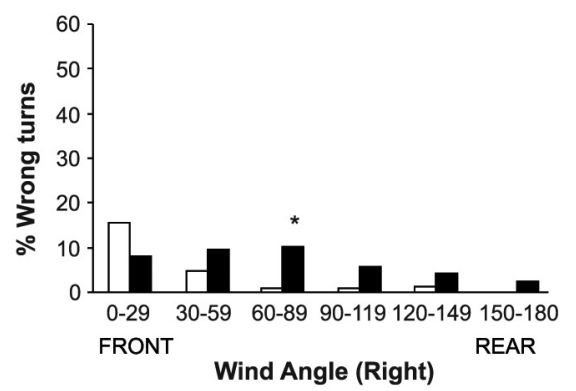

D Third-instar 'wrong' turns

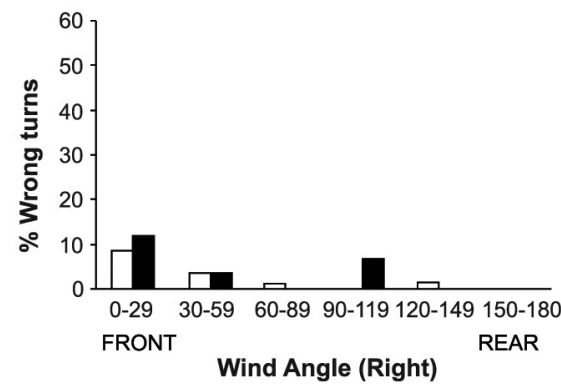

Figure 4. Engrailed knock-out significantly increases the proportion of toward turns but leaves wrong turns essentially unaffected. Percentage of different turn types according to the angle of the wind stimulus. Controls are indicated by open bars, and dsRNA-treated data are indicated by filled bars. $\boldsymbol{A}$, Second-instar toward turns. There is a significant increase in toward turns for winds from rear sectors. $\boldsymbol{B}$, Second-instar wrong turns. $\boldsymbol{C}$, Third-instar toward turns. $\boldsymbol{D}$, Third-instar wrong turns. $\chi^{2}$ test or Fisher's exact test: ${ }^{*} p<0.05 ;{ }^{* *} p<0.01 ;{ }^{* * *} p<0.001$.

sponses in both second and third instars (Fig. 3C,E). There are clearly a greater proportion of escapes in the upper part of the graphs that represent turns toward the right, that is, toward the wind stimulus. To describe this change in more detail, the relative proportions of toward and wrong turns were plotted as a function of wind direction (Fig. 4). In the second-instar control animals, few toward turns were made, but these were more frequent with wind stimuli from the rear (Fig. 4A). En knock-out caused a large increase in the frequency of this type of turn. The most striking example of this is that toward turns were made for $>52 \%$ of wind stimuli in the $150-180^{\circ}$ rear sector, rather than only $9 \%$ in controls. Third-instar animals showed very similar results (Fig. 4C). The results were less striking for wrong turns (turns that were so large or small that they moved the animal near to the wind source). In second- and third-instar controls, most wrong turns were made in response to wind from the front (Fig. $4 B, D$ ). En knock-out significantly increased the proportion of wrong turns only for wind from one $\left(60-89^{\circ}\right)$ sector (Fisher's exact test) in the second instar (Fig. 4B).

\section{Multipeak escape trajectories in juvenile cockroaches}

Additional insights into adult cockroach escape behaviors come from analyzing the escape trajectory, defined as the angle between the stimulus and the escape path of the animal (Fig. $5 A, B$ ) (Domenici et al., 2008). The ET is a circular distribution and allows the detection of multiple modes using circular statistics (Domenici and Blake, 1993; Domenici, 2002). ETs were calculated for second- and third-instar cockroaches, with and without dsRNA injection (Figs. 5, 6). The most striking feature of the control data is the multipeaked distribution of escape trajectories (Fig. 5C).
This was significantly different from a uniform circular distribution (Watson's $U^{2}$ test, $p<0.005$ ) and also from a single von Mises distribution (the circular version of a normal distribution). It is clear therefore that these multiple peaks are not simply a statistical artifact caused by wide variability. This test tells us that more than one peak is present; to determine the number and position of the peaks, it was necessary to use a more complex curve-fitting procedure. To determine quantitatively the angular position and the number of ET peaks, mixtures of multiple Gaussian curves were fitted to the unbinned data using the maximum likelihood method used for studies of the adult escape response (Domenici et al., 2008), using, as before, the constraints of equally spaced peaks with equal widths to minimize the number of parameters.

The second-instar control data were best fit by a multipeak distribution with five peaks that contributed at least 5\% to the distribution at 95, 122, 149, 175, and $202^{\circ}$, each with an SD of $9.1^{\circ}$. The Akaike weight of the fit (i.e., probability of being the correct one) was 0.73 . The relative contributions of each peak to the best-fit distribution were $5,12,30,41$, and $8 \%$, respectively (Fig. 5C). This distribution corresponds remarkably well to that found in a previous study of adult animals (Domenici et al., 2008), in which the ET peaks were located at 97, 124, 150,177 , and $204^{\circ}$, with a slightly smaller SD of $8.0^{\circ}$. This close correspondence between separate experiments is a convincing independent confirmation that these multiple ET peaks are real and not an artifact attributable to random variation.

\section{As in the adult, ET peaks depend on wind direction}

It was found in the adult study (Domenici et al., 2008) that the direction of the wind affects the "choice" of ETs: for wind from the posterior, ETs of 150,177 , and $204^{\circ}$ are used; for anterior wind, the $204^{\circ} \mathrm{ET}$ is not used, $177^{\circ}$ is used less frequently, and smaller ETs at $97^{\circ}$ and even $70^{\circ}$ come into play. The ET distribution for anterior wind thus has more peaks than that for posterior wind (Domenici et al., 2008).

We show here that these different strategies of ET usage are also present in the second-instar animals. ET data were divided into two categories: ETs in response to wind from the front quadrant $\left[0-89^{\circ}\right.$ wind direction (Fig. $\left.\left.5 D\right)\right]$ and those in response to wind from the rear quadrant $\left[90-180^{\circ}\right.$ wind direction (Fig. 5E)]. There are highly significant differences between the two distributions (Watson's $U^{2}$ test, $p<0.001$ ). Similar multipeak distributions were fitted to the front and rear ET populations, this time not changing the position or widths of the peaks but only optimizing the relative contribution of each Gaussian curve (Fig. $5 D, E)$. In this way, it was determined that, as in the adult, the relative contributions of the ET peaks to the overall distribution changed according to wind direction. In response to wind from the front, the $95,122,149,175$, and $202^{\circ}$ ET peaks made contributions of $10,17,33,32$, and $5 \%$, respectively, to the overall distribution, whereas, for rear wind, these ET peaks made relative 
contributions of 1 (too small to be shown on the figure), $6,26,52$, and $13 \%$. Thus, as in the adult, for wind from the front, there are several ET peaks, at large and small angles, whereas for rear wind, there are fewer peaks, at larger angles only.

En knock-out alters the distribution of escape trajectories to rear wind

En knock-out in second-instar animals has a highly significant effect on the ET distribution (Fig. $5 C$ vs $F$ ) (Watson's $U^{2}$ test, $p<0.001)$. Dissection into anterior and posterior distributions shows that only the $90-180^{\circ}$ (rear) ET distribution differs significantly from the control (Fig. 5H) (Watson's $U^{2}$ test, $p<0.001$ ). Thus, En knock-out affects the perception of rear wind only.

What is the exact nature of this change? The ET distributions for En knock-outs were not significantly different from single von Mises curves (Watson's $U^{2}$ test). This could reflect changes in contribution, or even widening, of the ET peaks themselves but could also be in part attributable to the lower numbers of experimental data. To determine how ET usage had changed, we compared the numbers of ETs in $30^{\circ}$ angular sectors that were centered on the approximate ET peaks. There were significant differences between En knock-outs and controls $\left(90-180^{\circ}\right)$ (Fig. $5 E, H$ ) for posterior wind only. Thus, in En knockouts, wind from the posterior of the animal elicits significantly fewer ETs in the $165-195^{\circ}$ sector (which includes the $175^{\circ}$ ET peak in controls) and more ETs in the 75-105, 105-135, and 135-165 sectors (which include the 95,122 , and $149^{\circ}$ peaks, respectively). The strategy for "choosing" ETs in response to posterior wind has therefore become statistically indistinguishable from the strategy for anterior wind. Thus, the choice of these smaller, anterior-appropriate ETs will result in the increased number of toward turns that we see in Figures 3 and 4.

\section{Third-instar ETs are very similar to second instar}

Very similar results were obtained for third-instar animals (Fig. 6). Thus, for controls, there was no significant change in the distribution of ETs between second and third instar (Watson's $U^{2}$ test). The third-instar ET distribution was best fit by a multipeak distribution with four peaks that contributed at least $5 \%$ to the distribution: at $122,148,175$, and $202^{\circ}$, each with an SD of $8.8^{\circ}$. The Akaike weight of the fit was 0.95 . The relative contributions of each peak to the best-fit distribution were 12, 29, 48, and $5 \%$, respectively (Fig. $6 A$ ). Similarly to second instar, there was a marked dependence of ET on the initial wind angle, with a more multipeaked choice of ETs with wind from the front (Fig. $6 B$ ), including the appearance of an ET peak at $95^{\circ}$. For wind from the
B

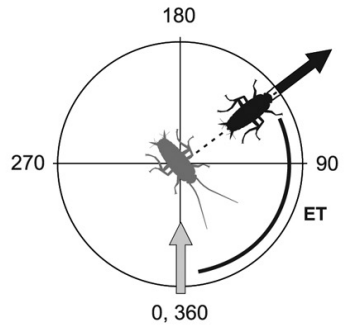

0,360
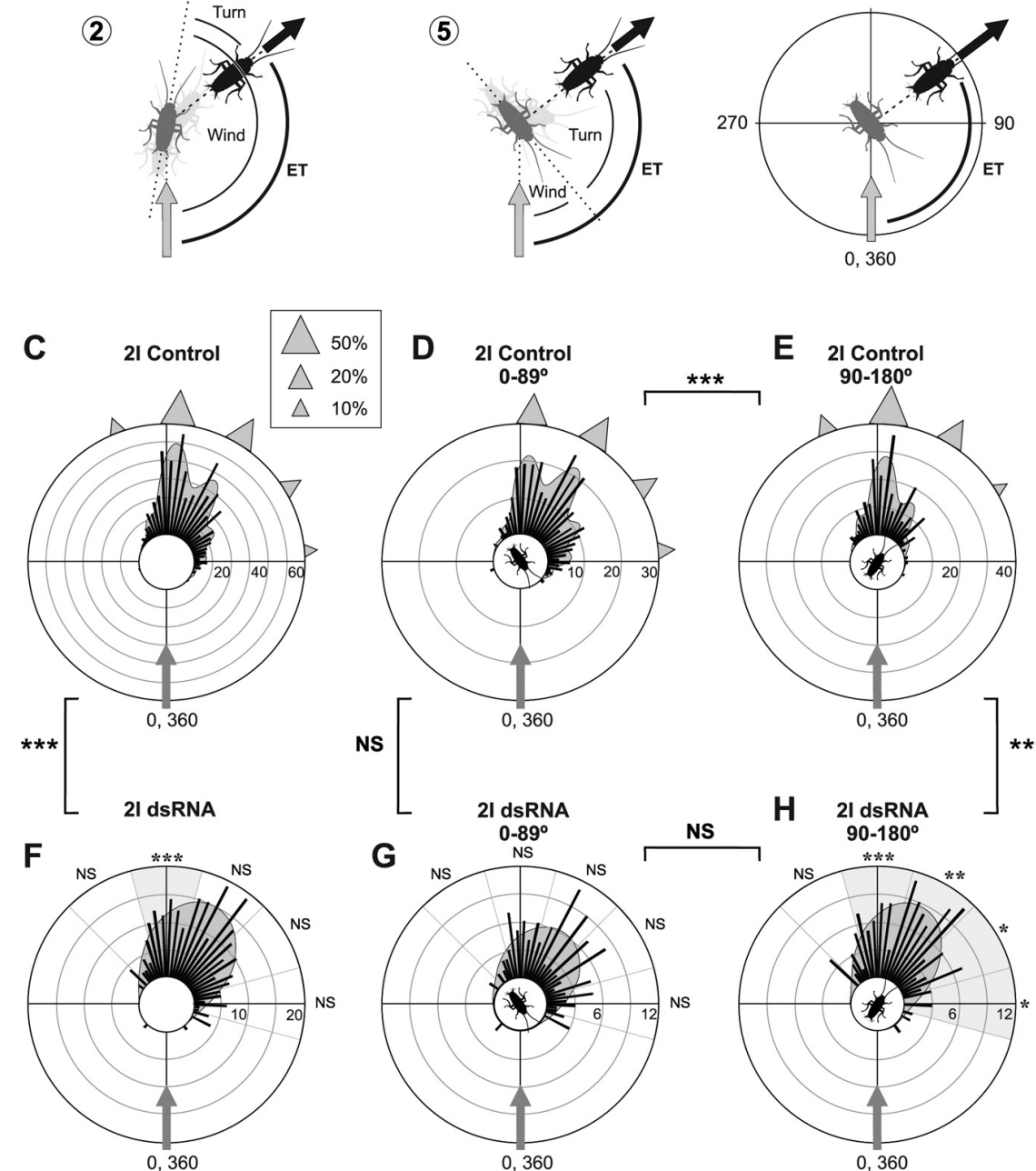

Figure 5. En knock-out significantly alters the distribution of escape trajectories. $\boldsymbol{A}$, Two diagrams of escape turns from Figure $3 A$ ( 2 and 5 ), with the stimuli aligned (gray arrows). Wind angle (wind), body turn (turn), and the corresponding escape trajectory (ET) are shown. Escape trajectory is the angle between wind direction (gray arrow) and the direction of motion (black arrow) of the escaping (black) cockroach. $\boldsymbol{B}$, Diagram illustrating the way ETs are plotted in subsequent figures. $\mathbf{C}-\boldsymbol{H}$, Polar frequency histograms of second-instar escape trajectories, with arrowheads indicating the positions of the five peaks in the best-fit ET distribution shown by the thin curve. The area of each arrowhead is proportional to the percentage contribution of that peak to the overal 列 $<5$ to the distribution. $\boldsymbol{F}$, Second-instar, En knock-out animals. A single peak adequately fits the data. There are sectors. $\boldsymbol{H}$, Second-instar En knock-out, wind from the rear quadrant. A single peak adequately fits the data, which is significantly different from controls in the $75-105,105-135,135-165$, and 165-195 sectors (light gray). $\chi^{2}$ test or Fisher's exact test: ${ }^{*} p<$ $0.05 ;{ }^{* *} p<0.01 ;{ }^{* *} p<0.001 ; \mathrm{NS}$, not significantly different.

rear (Fig. 6C), the ET distribution was significantly different (Watson's $U^{2}, p<0.001$ ), with a strong $(71 \%)$ preference for ETs of $175^{\circ}$.

As in the second instar, En knock-out also significantly altered the overall ET distributions of third-instar animals (Watson's $U^{2}$ test, $p<0.05)$. As with the second-instar animals, none of the dsRNA ET distributions were significantly different from single von Mises curves (Watson's $U^{2}$ test). Again, the ET distribution in response to wind from the posterior was significantly altered by dsRNAi, becoming wider than in controls, with significantly fewer ETs in the $165-195^{\circ}$ sector and more ETs in the 105-135 and $135-165^{\circ}$ sectors. 


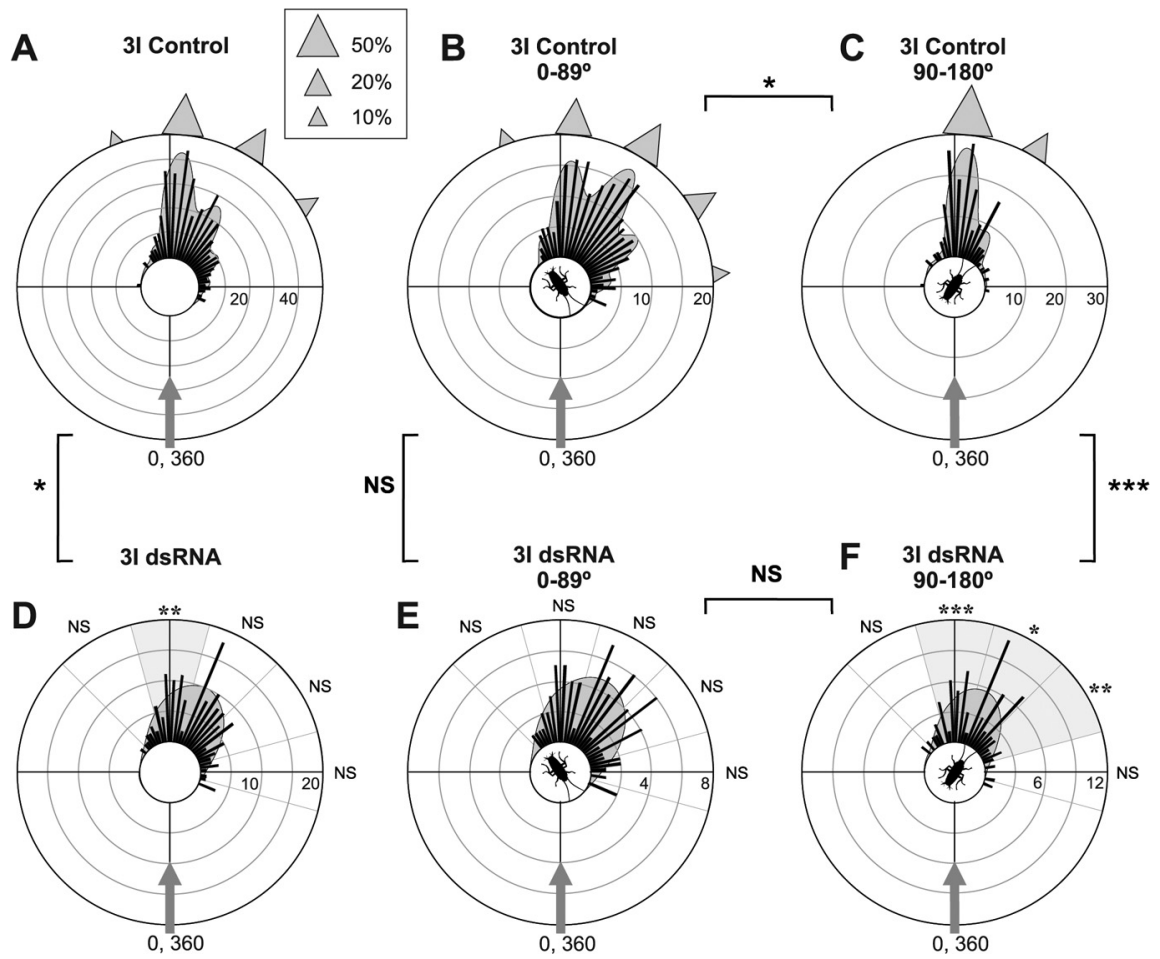

Figure 6. Persistent effect of En knock-out on the distribution of escape trajectories. Polar frequency histograms of third-instar escape trajectories, with arrowheads indicating the positions of the five peaks in the best-fit ET distribution shown by the thin curve. The area of each arrowhead is proportional to the percentage contribution of that peak to the overall distribution. $\boldsymbol{A}-\boldsymbol{C}$, Control animals. $\boldsymbol{D}-\boldsymbol{F}$, DsRNA-treated animals. $\boldsymbol{B}, \boldsymbol{E}$, Wind from the front quadrant $\left(0-89^{\circ}\right)$. $\boldsymbol{C}, \boldsymbol{F}$, Wind from the rear quadrant $\left(90-180^{\circ}\right) \cdot \chi^{2}$ test or Fisher's exact test: ${ }^{*} p<0.05 ;{ }^{* *} p<0.01$; ${ }^{* * *} p<0.001$; NS, not significantly different.

\section{Simulations predict that responses to wind from the rear are most affected by En knock-out}

We know that En knock-out alters the strengths of individual monosynaptic sensory inputs to GIs (Marie et al., 2000, 2002; Marie and Blagburn, 2003). How does this change in sensory input affect the directional sensitivities of the interneurons? In the adult cockroach, the three ventral GIs (vGIs) that are mainly responsible for triggering the escape response, GI1-GI3, have overlapping but distinct directional sensitivities. GI2 and GI3 respond mainly to wind from the lateral/rear and lateral/anterior quadrants, respectively, whereas GI1 responds mainly to stimuli from both front and rear lateral quadrants (Kolton and Camhi, 1995) (Fig. 7C).

In adult animals, the receptive fields of the GI can be measured directly by electrophysiological means (Miller et al., 1991; Kolton and Camhi, 1995) It is unfortunately not possible to measure the receptive fields of second- and third-instar GIs this way because the recording setup necessitates submersion of the cerci in saline. Instead, receptive fields were estimated using previously described computational methods (Hill and Blagburn, 1998). An extensive characterization of the synaptic inputs from cercal sensory neurons to GIs has been made (Thompson et al., 1992; Marie et al., 2000). It is therefore possible to combine the estimated directional responses of the sensory neurons with the strength of their synaptic connections to the GIs and, with this simulation, predict the directional sensitivities of the GIs in normal and En knock-out animals.

In contrast to the adult, the second-instar cerci are held almost vertically, rotated somewhat about their long axes (Fig. 7A). Every hair responds preferentially to wind from its lateral side, with the exception of the column " $h$ " hairs, which respond best in the reverse direction (Thompson et al., 1992). In the absence of any data for the second instar, we used the directional tuning curve for the first-instar medial sensory neuron (Dagan and Volman, 1982) in our simulation of these second-instar sensory neurons.

Using monosynaptic amplitude data based on previous studies (Thompson et al., 1992; Marie et al., 2000), we were able to estimate the total strength of synaptic input from each wind direction and, knowing the spike threshold of each GI, estimate the spiking frequency in the first $40 \mathrm{~ms}$ after a stimulus (Fig. 7B). This gives directional curves for the GIs that are very similar to those measured for the adult (Fig. 7C) (Kolton and Camhi, 1995), reinforcing our confidence in this computational approach.

In previous studies (Marie et al., 2000, 2002; Marie and Blagburn, 2003), En knock-out lead to the reduction in amplitude of existing synaptic connections from the medial sensory neuron $6 \mathrm{~m}$ to GI1 and GI2 of $\sim 30-50 \%$, whereas, more significantly, $6 \mathrm{~m}$ made novel connections to GI3 that are $\sim 60 \%$ the amplitude of control connections to GI2. We assumed that all En-expressing neurons in the cercus (indicated in Fig. $7 A$ by shaded wind tuning curves) would be similarly affected, with the exception of the neurons on the third and fourth segments that have already sent axons into the CNS at hatching (Marie et al., 2002) and of the column h neurons that only express small amounts of En (Blagburn et al., 1995). Changing the strengths of the synaptic amplitudes, to simulate En knock-out, led to relatively small changes in the directional tuning curves of GI1 and GI2, making them respond with one or two fewer spikes to rear wind (Fig. 7B). However, in the simulation, En knock-out led to a very marked change in the tuning curve of GI3, making it respond with up to five spikes to rear wind, whereas normally it would be silent (Fig. 7B).

How would these changes affect the turning responses of the animal? The proposed effects are illustrated qualitatively in Figure $8 \mathrm{~A}$, with shading illustrating the changing probability of ET usage with wind angle that we have demonstrated previously in the adult insect (Domenici et al., 2008) and in the current study of second- and third-instar control cockroaches. The predicted effects of En knock-out would be twofold. First, the diagonal ET bands are distorted for the responses to rear wind (Fig. $8 \mathrm{~B}$ ); the turns are somewhat larger than they should be because of increased synaptic input to both of the GI3 interneurons. The actual degree of curvature could be greater or lesser than that depicted, depending on how exactly the changes in synaptic inputs affect the computation of turn angle. This curvature of the ET bands would result in the "blurring" of ET peaks in the circular data plots of Figures 5 and 6, thus partially explaining why they are no longer detectable. The second, and more dominant, effect of En knock-out would be to change the strategy for using the different ETs; thus, small ETs are erroneously used for large wind angles, thereby accounting for the large increase in toward turns (indicated by the dashed oval). This change in ET usage would 

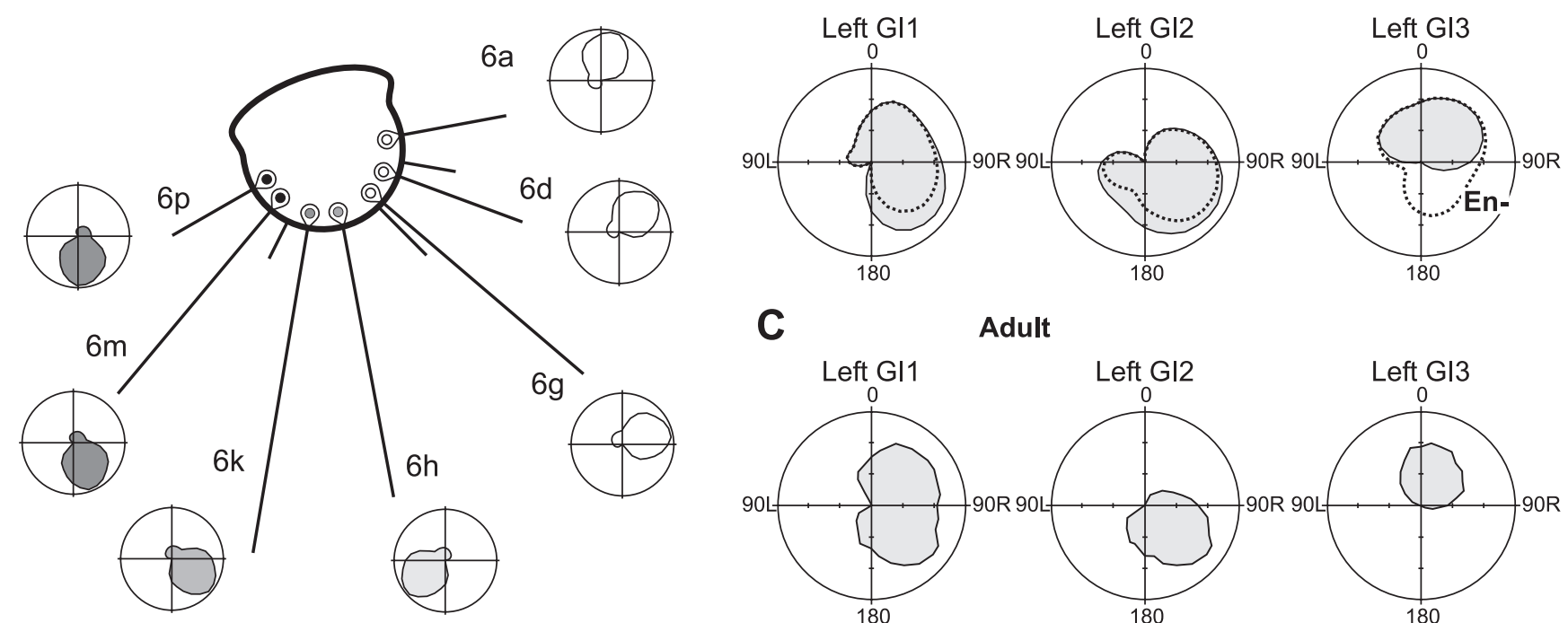

C

Adult

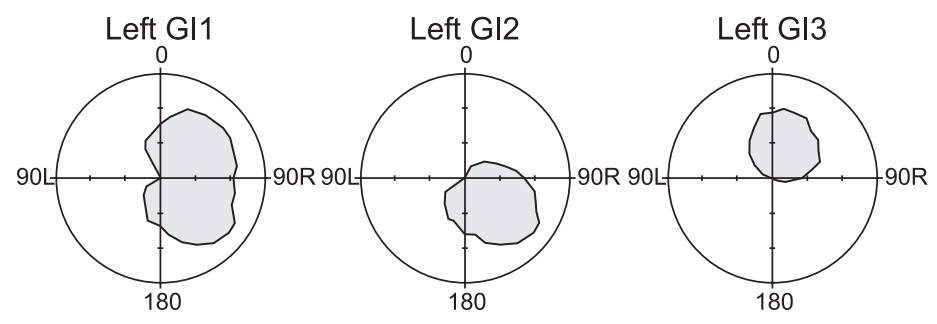

Figure 7. Simulation predicts that second-instar $\mathrm{Gl}$ receptive fields are very similar to the adult $\mathrm{Gl}$ receptive fields, which have been determined experimentally. $A$, Diagram of a transverse section of the right cercus, at the level of segment 6 , oriented with anterior to top, lateral to right. Hairs are shown approximately to scale. Hairs used in the simulation (those $>50 \mu \mathrm{m}$ in length) are indicated, along with an approximated receptive field. Receptive fields are polar plots of wind angle versus spike frequency, with the limit of the plot corresponding to $350 \mathrm{~Hz}$. En expression in the sensory neuron is indicated by shading of the cell nucleus and of the receptive field of the neuron. $B, C$, Receptive fields of the left ventral Gls, GI1-GI3. These Gls have their axons and main dendritic fields on the right side of the animal. $\boldsymbol{B}$, Second-instar receptive fields, calculated by combining the sensory-hair receptive fields with the known synaptic amplitudes and spike thresholds. The plot boundary indicates nine action potentials within the summation time of $40 \mathrm{~ms}$. The receptive field estimated for normal animals is indicated in gray, the En dsRNA-treated receptive field by a bold dotted line. C, Receptive fields of GI1-GI3 in the adult, adapted from Kolton and Camhi (1995).

tend to mask any moderate increase in turn size, explaining why we see no increase in mean turn angle.

\section{Discussion}

\section{En knock-out alters the response to posterior wind}

In previous work, we have shown that En knock-out alters the synaptic connections made by second-instar cockroach filiform hair sensory neurons onto GIs (Marie et al., 2000, 2002; Marie and Blagburn, 2003). We have also shown that adult cockroaches use multiple escape trajectories with probabilities that differ according to wind direction (Domenici et al., 2008). Here we put those results together and show that second- and third-instar larval cockroaches use the same ETs as the adult and show the same strategies of ET usage for anterior versus posterior wind. We also show that En knock-out apparently results in a change in perception of posterior wind, evidenced by the use of an ET strategy more appropriate for stimulation from the front. This "anterior strategy" involves the use of more small-angle ETs and so explains why we see more toward turns in response to posterior wind. These results are consistent with the previously observed changes in synaptic connectivity.

\section{The central origin of the ET peaks}

How, and where, do the multiple ETs arise? It has been established previously that, because the distribution of wind angles is uniform and ET is the sum of wind angle plus the body turn, the multimodal ETs cannot be attributable to any mechanical or neural constraints that restrict the cockroach to performing turns only at certain fixed angles relative to its body, nor can they be attributable to measurement artifacts (Domenici et al., 2008). The present study provides strong additional evidence that ET peaks cannot be artifactual because they are almost identical in size and position in second- and third-instar larvae and adults.

This conservation of the ET-generating mechanism (whatever it is) throughout several months of postembryonic development strongly implies that the mechanism that generates the ETs is located in the CNS rather than being an emergent property of the sensory input. As is typical of hemimetabolous insects, the sensory complement continues to grow as body size increases, whereas the number of CNS neurons remains the same (Gymer and Edwards, 1967). Thus, there is a large increase in sensory hair numbers from 39 per cercus in the second instar to 220 in the adult. Although the ET peaks themselves are therefore unlikely to be generated by the sensory input, it is possible that having fewer sensory neurons could theoretically decrease the angular resolution of wind detection; a lower resolution would then increase the SD of the ET peaks. However, the large difference in numbers of sensory hairs between adult (220 hairs) and second instar (39 hairs) clearly does not have a commensurate blurring effect on the SD of the ET peaks $\left(8.0 \mathrm{vs} 9.1^{\circ}\right)$. The possibility remains that, in the first instar, with only two hairs on each cercus, the ET peaks would appear wider or not distinguishable at all, even if the central ET-generating mechanism were identical.

\section{Neural mechanisms of ET generation}

It is thought that the three vGIs, GI1-GI3, are primarily responsible for mediating the escape response, whereas the four dorsal GIs play a role during flying (Kolton and Camhi, 1995). In the thorax, the ventral GIs synapse with the TIa group of interneurons (Ritzmann and Pollack, 1988; Westin et al., 1988), which contact leg motor neurons directly, and via local interneurons (Ritzmann and Pollack, 1990). However, the measured wind fields of the vGIs and TIa interneurons are rather extensive. Although it appears to be possible for an angular resolution at least as small as $20^{\circ}$ to be encoded by a vGI (Levi and Camhi, 2000b), the resolution necessary to achieve the narrow ET peaks we describe here (and previously) may require the involvement of other interneurons. These may include the dorsal GIs (GI4-GI7) and other, as yet unidentified, intersegmental interneurons. In 
A

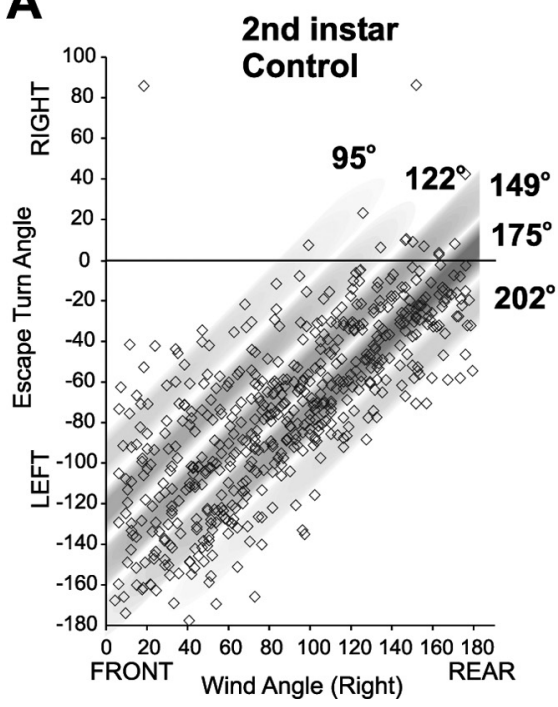

B

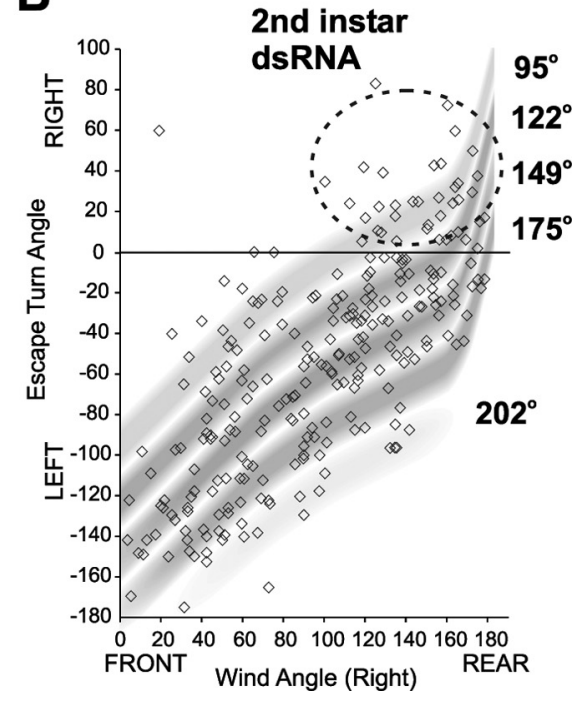

knock-out will increase the strength of synaptic input from medial hairs onto the left and right GI3s, thus making them respond to wind from the rear as well as the front (Kolton and Camhi, 1995). It is known that increasing the number of action potentials in the axon of a single GI3 causes an increase in turning tendency away from the side of the animal on which the axon is located (Levi and Camhi, 2000b). Therefore, we predicted that turn size in response to rear wind should increase somewhat, although less, perhaps, than would be expected if the activity of a single GI3 were increased. More importantly, we also predicted that the increased activation of both GI3s by En knock-out leads to the more frequent recruitment of small-angle ETs that, at large wind angles, result in turns toward the same side as the stimulus.

The implication of the previous study on adult animals was that ETs are generated by a geocentric mechanism, i.e., in which the trajectories are fixed relative to an external reference (the stimulus) rather than by an egocentric mechanism in which trajectories are generated relative to a body

the thorax, the axons of these neurons are known to activate the directionally sensitive TIb interneurons (Westin et al., 1988).

Previous work has not explained why so many $(\sim 100)$ thoracic interneurons are required for the cockroach escape response. Some of them probably coordinate the different movements required of each leg in response to wind from different directions, whereas others may mediate proprioceptive reflexes that also affect the degree of leg movement (Nye and Ritzmann, 1992). However, a computational model of the cockroach escape circuit showed that, in theory, only two excitatory and two inhibitory interneurons in each thoracic ganglion are needed to generate a facsimile of the directional aspects of the escape behavior, although, of course, the existence of multiple ETs could not be taken into account (Ezrachi, 2003). One possibility suggested by our work is that subpopulations of these numerous thoracic interneurons could be responsible for generating the different ETs. In this case, we would expect that fewer of these interneuronal groups would be activated by spikes in GI2 (signifying wind from the back), leading to only the three larger ETs being used, whereas spikes in GI3 (wind from the front) would potentially activate more groups and lead to a wider choice between the four smaller ETs.

There is of course the additional possibility that descending inputs from the brain and/or subesophageal ganglion could also be involved in generating the ETs, because lesions rostral to the thorax change the orientation of the initial turning component and shorten the duration of the escape run (Keegan and Comer, 1993).

\section{Effects of En knock-out on ETs: geocentricity versus egocentricity}

En knock-out alters the response to wind from the rear, increasing the number of small-angle ETs so that the ET distribution is similar to that for anterior wind. Our modeling of second-instar GI receptive fields seems to explain this by predicting that En reference (i.e., by fixed angles of body turn) (Domenici et al., 2008). The present results serve to strengthen that conclusion; if the ETs were generated by an egocentric mechanism, we would expect increased input to GI3 to simply increase turn angle, but this is not the case. Instead, En knock-out leads to a misperception of wind from the rear, and the animals geocentrically adopt anterior-appropriate ETs, which often result in smaller turns toward the stimulus.

Only in a few systems have transcription factors been shown to control synaptic connectivity and thereby influence behavior (Chen et al., 2003; Von Stetina et al., 2007; Clyne and Miesenböck, 2008; Shimizu et al., 2008; Yamamoto, 2008). We show here for the first time that knock-out of the transcription factor Engrailed, which alters the specificity of synaptic connections, can result in a change in perception of a directional stimulus. In the future, it will be of great interest to investigate this further at the molecular and cellular levels; to identify the downstream targets of Engrailed transcriptional regulation and to determine the cellular basis behind the generation of multiple escape trajectories.

\section{References}

Akaike H (1974) A new look at the statistical model identification. IEEE Trans Automat Contr AC-19:716-723.

Baader SL, Vogel MW, Sanlioglu S, Zhang X, Oberdick J (1999) Selective disruption of "late onset" sagittal banding patterns by ectopic expression of Engrailed-2 in cerebellar Purkinje cells. J Neurosci 19:5370-5379.

Batschelet E (1981) Circular statistics in biology. London: Academic.

Blagburn JM, Beadle DJ, Sattelle DB (1984) Synapses between an identified giant interneurone and a filiform hair sensory neurone in the terminal ganglion of first instar cockroaches (Periplaneta americana L.). J Exp Biol 113:477-481.

Blagburn JM, Gibbon CR, Bacon JP (1995) Expression of engrailed in an array of identified sensory neurons: comparison with position, axonal arborization, and synaptic connectivity. J Neurobiol 28:493-505.

Chen HH, Hippenmeyer S, Arber S, Frank E (2003) Development of the monosynaptic stretch reflex circuit. Curr Opin Neurobiol 13:96-102. 
Clyne JD, Miesenböck G (2008) Sex-specific control and tuning of the pattern generator for courtship song in Drosophila. Cell 133:354-363.

Comer CM, Dowd JP (1987) Escape turning behaviour of the cockroach. Changes in directionality induced by unilateral lesions of the abdominal nervous system. J Comp Physiol A Neuroethol Sens Neural Behav Physiol 160:571-583.

Condron BG, Patel NH, Zinn K (1994) Engrailed controls glial/neuronal cell fate decisions at the midline of the central nervous system. Neuron 13:541-554.

Dagan D, Camhi JM (1979) Responses to wind recorded from the cercal nerve of the cockroach Periplaneta americana. II. Directional sensitivity of sensory axons innervating single columns of filiform hairs. J Comp Physiol A Neuroethol Sens Neural Behav Physiol 133:103-111.

Dagan D, Volman SF (1982) Sensory basis for directional wind detection in first instar cockroaches, Periplaneta americana. J Comp Physiol A Neuroethol Sens Neural Behav Physiol 147:471-478.

Domenici P (2002) Escape trajectory, ecological. In: Encyclopedia of environmetrics (El-Shaarawi AH, Piegorsch WW, eds), pp 708-711. Chichester, UK: Wiley.

Domenici P, Blake RW (1993) Escape trajectories in angelfish (Pterophyllum eimekei). J Exp Biol 177:253-272.

Domenici P, Booth D, Blagburn JM, Bacon JP (2008) Cockroaches keep predators guessing by using preferred escape trajectories. Curr Biol 18:1792-1796.

Ezrachi EA (2003) Computational model of the cockroach escape behavior: winner and losers in a population code. Biol Cybern 88:33-45.

Friedman GC, O’Leary DD (1996) Retroviral misexpression of engrailed genes in the chick optic tectum perturbs the topographic targeting of retinal axons. J Neurosci 16:5498-5509.

Gymer A, Edwards JS (1967) The development of the insect nervous system. I. An analysis of postembryonic growth in the terminal ganglion of Acheta domesticus. J Morphol 123:191-197.

Heemskerk J, DiNardo S, Kostriken R, O’Farrell PH (1991) Multiple modes of engrailed regulation in the progression towards cell fate determination. Nature 352:404-410.

Hill ES, Blagburn JM (1998) Indirect synaptic inputs from filiform hair sensory neurons contribute to the receptive fields of giant interneurons in the first-instar cockroach. J Comp Physiol A Neuroethol Sens Neural Behav Physiol 183:467-476.

Holman D (2003) mle: a programming language for building likelihood models. Version 2.1, Vol 1, User's manual. Vol 2, Reference manual. http://faculty.washington.edu/ djholman/mle.

Hurvich C, Tsai C (1989) Regression and time series model selection in small samples. Biometrika 78:499-509.

Joly W, Mugat B, Maschat F (2007) Engrailed controls the organization of the ventral nerve cord through frazzled regulation. Dev Biol 301:542-554.

Keegan AP, Comer CM (1993) The wind-elicited escape response of cockroaches (Periplaneta americana) is influenced by lesions rostral to the escape circuit. Brain Res 620:310-316.

Kolton L, Camhi JM (1995) Cartesian representation of stimulus direction: parallel processing by two sets of giant interneurons in the cockroach. J Comp Physiol A Neuroethol Sens Neural Behav Physiol 176:691-702.

Levi R, Camhi JM (2000a) Population vector coding by the giant interneurons of the cockroach. J Neurosci 20:3822-3829.

Levi R, Camhi JM (2000b) Wind direction coding in the cockroach escape response: winner does not take all. J Neurosci 20:3814-3821.

Logan C, Wizenmann A, Drescher U, Monschau B, Bonhoeffer F, Lumsden A
(1996) Rostral optic tectum acquires caudal characteristics following ectopic engrailed expression. Curr Biol 6:1006-1014.

Lundell MJ, Chu-LaGraff Q, Doe CQ, Hirsh J (1996) The engrailed and huckebein genes are essential for development of serotonin neurons in the Drosophila CNS. Mol Cell Neurosci 7:46-61.

Marie B, Bacon JP (2000) Two engrailed-related genes in the cockroach: cloning, phylogenetic analysis, expression and isolation of splice variants. Dev Genes Evol 210:436-448.

Marie B, Blagburn JM (2003) Differential roles of Engrailed paralogs in determining sensory axon guidance and synaptic target recognition. J Neurosci 23:7854-7862.

Marie B, Bacon JP, Blagburn JM (2000) Double-stranded RNA interference shows that Engrailed controls the synaptic specificity of identified sensory neurons. Curr Biol 10:289-292.

Marie B, Cruz-Orengo L, Blagburn JM (2002) Persistent engrailed expression is required to determine sensory axon trajectory, branching, and target choice. J Neurosci 22:832-841.

Miller JP, Jacobs GA, Theunissen FE (1991) Representation of sensory information in the cricket cercal sensory system. I. Response properties of the primary interneurons. J Neurophysiol 66:1680-1689.

Nüsslein-Volhard C, Wieschaus E (1980) Mutations affecting segment number and polarity in Drosophila. Nature 287:795-801.

Nye SW, Ritzmann RE (1992) Motion analysis of leg joints associated with escape turns of the cockroach, Periplaneta americana. J Comp Physiol A Neuroethol Sens Neural Behav Physiol 171:183-194.

Ritzmann RE, Pollack AJ (1988) Wind-activated thoracic interneurons of the cockroach. II. Patterns of connection from ventral giant interneurons. J Neurobiol 19:589-611.

Ritzmann RE, Pollack AJ (1990) Parallel motor pathways from thoracic interneurons of the ventral giant interneuron system of the cockroach, Periplaneta americana. J Neurobiol 21:1219-1235.

Saueressig H, Burrill J, Goulding M (1999) Engrailed-1 and netrin-1 regulate axon pathfinding by association interneurons that project to motor neurons. Development 126:4201-4212.

Shimizu H, Shimoda M, Yamaguchi T, Seong KH, Okamura T, Ishii S (2008) Drosophila ATF-2 regulates sleep and locomotor activity in pacemaker neurons. Mol Cell Biol 28:6278-6289.

Siegler MV, Jia XX (1999) Engrailed negatively regulates the expression of cell adhesion molecules connectin and neuroglian in embryonic Drosophila nervous system. Neuron 22:265-276.

Stern M, Ediger VL, Gibbon CR, Blagburn JM, Bacon JP (1997) Regeneration of cercal filiform hair sensory neurons in the first-instar cockroach restores escape behavior. J Neurobiol 33:439-458.

Thompson KS, Blagburn JM, Gibbon CR, Bacon JP (1992) Correlation of filiform hair position with sensory afferent morphology and synaptic connections in the second instar cockroach. J Comp Neurol 320:213-227.

Von Stetina SE, Fox RM, Watkins KL, Starich TA, Shaw JE, Miller DM 3rd (2007) UNC-4 represses CEH-12/HB9 to specify synaptic inputs to VA motor neurons in C. elegans. Genes Dev 21:332-346.

Westin J, Langberg JJ, Camhi JM (1977) Responses of the giant interneurons of the cockroach Periplaneta americana to wind puffs of different directions and velocities. J Comp Physiol A Neuroethol Sens Neural Behav Physiol 121:307-324.

Westin J, Ritzmann RE, Goddard DJ (1988) Wind-activated thoracic interneurons of the cockroach. I. Responses to controlled wind stimulation. J Neurobiol 19:573-588.

Yamamoto D (2008) Brain sex differences and function of the fruitless gene in Drosophila. J Neurogenet 15:1-24. 